D) Check for updates

Cite this: Polym. Chem., 2019, 10, 1357

Received 29th October 2018 Accepted 26th January 2019 DOI: $10.1039 /$ c8py01540f rsc.li/polymers

\section{Enhanced reduction of polymerization-induced shrinkage stress via combination of radical ring opening and addition fragmentation chain transfer $\dagger$}

\author{
Sebastian Schoerpf, (DD a,b Yohann Catel, (ID) b,c Norbert Moszner, (ID) b,c \\ Christian Gorsche (DD *a,b and Robert Liska (D) ${ }^{a, b}$
}

Polymerization-induced shrinkage stress within bulk photopolymer networks represents one of the most pressing challenges for their application in 3D-printing, microelectronics and dentistry. The occurring shrinkage stress within commercial (meth)acrylate-based networks results from a combination of the covalent attachment of monomers and the gelation at early stages of the reaction. Alternatively, cyclic monomers (e.g. 1,1-disubstituted 2-vinylcyclopropanes, VCPs) have been reported as a possible monomer class yielding reduced polymerization shrinkage and consequently shrinkage stress due to a radical ring opening reaction. Another way to reduce the occurring shrinkage stress is to regulate the radical network formation via an addition fragmentation chain transfer (AFCT) concept. Herein, the lightinduced radical polymerization of a combination of VCP monomers with variable amounts of an AFCT reagent (an ester-activated vinyl sulfonate ester EVS) was investigated. A high reactivity towards lightinduced radical polymerization is confirmed for VCP/EVS mixtures via photoreactor, photo-DSC and real time (RT)-NIR-photorheology accompanied by a significant increase in $\mathrm{C}=\mathrm{C}$ double bond conversion with increasing amounts of EVS. Most importantly, both systems (VCPs and AFCT reagents) combined lead to an enhanced reduction of polymerization-induced shrinkage stress. The resulting materials showed, even at low concentrations of EVS, a high network homogeneity (indicated by a narrow loss factor plot in DMTA). Also, filled systems performed well with respect to reduced shrinkage force while maintaining sufficient E-modulus and flexural strength. The presented material concept has great potential for dental materials and lithography-based 3D-printing.

\section{Introduction}

Photoinitiated polymerizations of (meth)acrylate systems have been known for more than a half century. ${ }^{1}$ These types of formulations suffer from shrinkage stress, which turns out to be a restricting factor in various applications (e.g. coatings, ${ }^{2}$

\footnotetext{
${ }^{a}$ Institute of Applied Synthetic Chemistry, Technische Universität Wien, Getreidemarkt 9/163 MC, A-1060 Vienna, Austria.

E-mail: christian.gorsche@tuwien.ac.at

${ }^{b}$ Christian Doppler Laboratory for Photopolymers in Digital and Restorative Dentistry, Technische Universität Wien, Getreidemarkt 9/163 MC, A-1060 Vienna, Austria

${ }^{c}$ Ivoclar Vivadent AG, Bendererstrasse 2, FL-9494 Schaan, Liechtenstein $\dagger$ Electronic supplementary information (ESI) available: Described syntheses of ADVCP and TEGDVCP; photo-DSC plots and tables; RT-NIR-photorheology plots; storage stability plots; DMTA table, tensile test table, shrinkage force measurement of composite formulations table; mechanical properties table. See DOI: 10.1039/c8py01540f
}

3D-printing,,$^{3,4}$ microelectronics ${ }^{5,6}$ and dentistry $\left.{ }^{7,8}\right)$. Due to the inhomogeneous network architecture, which is created during the radical curing process, the resulting materials tend to show rather brittle behavior and the occurring shrinkage stress could lead to delamination, deformation or mechanical failure of the final bulk materials.

The observed shrinkage stress evolves during the polymerization reaction upon transitioning of the applied formulation from the liquid to solid state (i.e. gel point) and is built up upon vitrification until the final conversion is reached. Before polymerization the monomers are situated at van der Waals distance toward each other (approximately $3.4 \AA$ ). The occurring shrinkage stress upon gelation is in part due to the formation of covalent bonds between the respective monomers, where the revealing distance is only $1.5 \AA^{9} .^{9}$ This volumetric shrinkage of the material leads to stress, which cannot be dissipated within a vitrified matrix. The shrinkage stress after polymerization can often be observed as curl and creep distortion of the sample. ${ }^{3}$ 
A number of different strategies for the reduction of polymerization-induced shrinkage stress have been reported. By reducing the amount of reactive groups within the volume of the polymerizable matrix (e.g. introduction of inorganic fillers, ${ }^{9}$ nanogels ${ }^{10-12}$ the observed shrinkage stress is minimized. Stansbury has implemented nanogel-modified systems as promising candidates to reduce polymerization-induced shrinkage stress.

Another option is to redesign the monomers and the underlying curing mechanism. Replacing methacrylates (MAs) by cyclic monomers such as vinylcyclopropanes (VCPs), cyclic ketene acetals, spiro orthoesters or carbonates will lead to a reduction of shrinkage stress. ${ }^{13}$ Focusing on VCPs, the reduction of the shrinkage stress has been initially shown with 1,1-disubstituted 2-vinylcyclopropanes. ${ }^{14}$ In order to obtain polymer networks, multifunctional vinylcyclopropanes are required. Contreras et al. synthesized a replacement structure for a commonly-used urethane dimethacrylate (UDMA) containing VCPs, which showed a high reactivity in combination with a germanium-based visible-light photoinitiator (bis-4-(methoxybenzoyl)diethylgermanium BMDG). ${ }^{15}$ Ensuing studies by our research group have confirmed this high reactivity and a series of VCPs has been introduced as reactive diluents for composite formulations. ${ }^{16}$ By using BMDG as radical photoinitiator it was possible to generate highly reactive VCPbased formulations and composites with good mechanical properties. ${ }^{16}$ Moreover, VCPs bearing urethane groups lead to exceptionally low values of shrinkage stress. ${ }^{13}$

Another well-established approach to counteract polymerization-induced shrinkage stress are thiol-ene ${ }^{17}$ or thiol-yne $e^{18,19}$ systems. They undergo an alternating chain transfer mechanism, which leads to a shift of the gelation toward higher conversions and as a result to a reduction of the polymerizationinduced shrinkage stress. Thiol-ene chemistry is a good approach to obtain polymers with defined network architectures. Although thiol-ene reactions are highly efficient they are limited in the stability of the final formulation, ${ }^{20}$ often exhibit objectionable odor ${ }^{21}$ and they commonly suffer from softness and low $T_{\mathrm{g}}$ because of their thio-ether bridges, ${ }^{22}$ which has motivated research to strive for alternative solutions. Alternatively, addition fragmentation chain transfer (AFCT) reagents $^{23,24}$ can be applied to obtain a well-defined network structure of the final photopolymers. The addition of AFCT reagents is known as a versatile method to provide step growth-like characteristics to a radical polymerization. Moreover, $\beta$-allyl sulfides have already been tested for the regulation of polymer networks. ${ }^{25}$ Furthermore, the use of esteractivated $\beta$-allyl sulfones (EASs) has been demonstrated to generate more homogenous photopolymer networks with tailored (thermo)mechanical properties. ${ }^{26}$ These transfer agents show exceptional reactivity in methacrylate systems. This high reactivity with methacrylates results in improved monomer conversions, reduced shrinkage stress and high mechanical toughness. ${ }^{27}$ Via the regulation of the radical curing mechanism, the gel point is shifted to higher conversions. Thereby, it is possible to generate less shrinkage stress within the final polymer network and reach higher double bond (DB) conversions. In addition the (thermo)mechanical properties of the formed polymer can be tailored through content and functionality of the introduced AFCT reagent. While also having the option to enable network rearrangement or even flow of the network after gelation via integration of a reversible AFCT unit (e.g. $\beta$-allyl sulfide/thiol system) into the photopolymer network, ${ }^{28}$ one apparent drawback of most AFCT reagents is the concomitant retardation of the polymerization process. Recently, an ester-activated vinyl sulfonate ester (EVS) has been reported as powerful AFCT reagent showing no retardation during the radical polymerization process. ${ }^{29}$ This improved curing behavior has enabled the 3D-structuring of such toughened photopolymers with low shrinkage stress.

Herein, we examined the combination of VCPs, which undergo a RROP during curing, with AFCTs, which generate a more homogenous network structure and subsequently shift gelation toward higher conversions. This should allow an even further reduction of polymerization-induced shrinkage stress. Generally, VCPs perform a radical ring opening polymerization (RROP, Fig. 1) and according to the RROP the propane ring opens and a tertiary radical is formed (INT1 $1^{\circ}$ ), which can attack another double bond (DB). In addition, a minor amount of the radicals perform a backbiting step in which a cyclobutane radical is formed $\left(\mathrm{CB}^{\circ}\right)$. This step is not limiting the reaction as the radical can further attack another DB and continue radical propagation (P). Additionally, via the implementation of an AFCT reagent (e.g. EVS) a chain transfer (CT) step will be forced by the attack of a radical on EVS. This attack leads to the intermediate radical (INT2 ${ }^{\circ}$ ), which fragments irreversibly to a carbonyl group and a highly reactive tosyl radical $\left(\mathrm{L}^{\circ}\right)$.

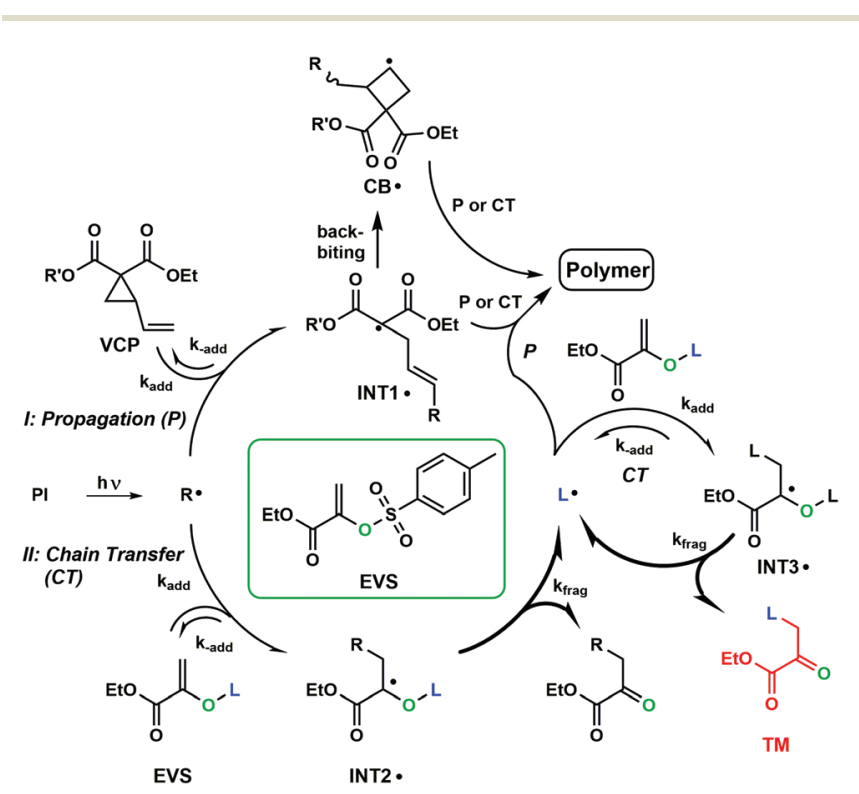

Fig. 1 AFCT mechanism of an ester-activated vinyl sulfonate ester (EVS) in a photoinitiated radical polymerization with vinylcyclopropanes (VCPs); ( $\mathrm{L}$ - Tos; $\mathrm{R}$ - from photoinitiator cleavage or propagating radical; $\mathrm{R}^{\prime}$ - R-group of the respective VCP derivative). 
This mechanism prevents the reaction from retardation by favoring fragmentation. During the curing process initiator radicals as well as the generated tosyl radical $\left(\mathrm{L}^{\circ}\right)$ attack VCPs and further form propagating radical moieties, which crosslink to a network in multifunctional systems. The attack of a tosyl radical $\left(\mathrm{L}^{\circ}\right)$ on another EVS molecule (INT3*) and subsequent fragmentation would lead to an unwanted side product (TM). However, this self-reaction is kinetically not favored and unlikely to occur in formulations containing $<50 \%$ of EVS. ${ }^{29}$

In an unregulated MA-based network the gel point is reached very fast and at low conversions, typically below $20 \%$. Resulting networks suffer from high shrinkage stress (Fig. 2a). To reduce the shrinkage stress of a polymerizing network the MAs can be replaced by VCPs. VCPs undergo RROP, which results in lower volumetric shrinkage that ultimately yields reduced shrinkage stress with the polymerized material (Fig. 2b). Through the addition of an AFCT reagent to a MA system the gel point can be shifted toward higher conversions, thus significantly reducing the occurring shrinkage stress (Fig. 2c). By combining VCPs with a transfer agent we postulate an enhanced reduction of shrinkage stress via both concepts, RROP and shifting the gel point with AFCT reagents toward higher conversions (Fig. 2d).

\section{Experimental section}

\section{Materials and general methods}

Diethyl 1,1'-(((2,2,4-trimethylhexane-1,6-diyl)bis(azanediyl))bis (carbonyl))bis(2-vinyl-cyclopropane-1-carboxylate) (ADVCP), ${ }^{15}$ the O'1,O1-((ethane-1,2-diylbis(oxy)) bis(ethane-2,1-diyl)) 1,1'diethyl bis(2-vinylcyclopropane-1,1-dicarboxylate) (TEGDVCP) ${ }^{16}$ and the ester activated vinyl sulfonate (EVS) ${ }^{30}$ were synthesized according to previous literature methods.

The monofunctional VCP diethyl 2-vinylcyclopropane-1,1dicarboxylate (DVD) and the photoinitiator bis(4-methoxybenzoyl)diethylgermane (BMDG) were kindly provided by the company Ivoclar Vivadent AG. Dichloromethane (DCM) was dried using a PureSolv system (inert technology, Amesbury, MA, USA). $N, N^{\prime}$-Dicyclohexylcarbodiimide (DCC), 1-ethyl-3(3-dimethylaminopropyl)carbodiimide (EDC.HCl), butylhydroxytoluene (BHT), phenothiazine, 2,2,4(2,4,4)-trimethyl-1,6hexanediamine and triethylene glycol were purchased from Sigma-Aldrich and used as received. ${ }^{1} \mathrm{H}-\mathrm{NMR}$ spectra were recorded on a Bruker Avance at $400 \mathrm{MHz}\left(100 \mathrm{MHz}\right.$ for $\left.{ }^{13} \mathrm{C}\right)$; chemical shifts are given in ppm and were referenced to the solvent residual peak $\left(\mathrm{CDCl}_{3}\right)$. Multiplicities are referred to as $\mathrm{s}$ (singlet), d (doublet), t (triplet), q (quartet) and m (multiplet). Coupling constants are given in $\mathrm{Hz}$.

\section{Resin formulations}

The vinylcyclopropane monomers ADVCP and TEGDVCP were mixed in a $7 / 3$ molar ratio (VCP), EVS was added in $5 \mathrm{DB} \%$ (VCP/EVS_5), 10 DB\% (VCP/EVS_10), 15 DB\% (VCP/EVS_15) and $20 \mathrm{DB} \%$ concentration (VCP/EVS_20; DB\% - molar percentage of reactive double bonds within a formulation). A methacrylate reference MA was prepared with the monomers UDMA and TEGDMA, which were mixed in a 7/3 molar ratio. The VCP and methacrylate reference contained no AFCT reagent. For the performed photo-DSC, RT-NIR-photorheology measurements, DMTA, tensile tests and storage stability tests, $0.5 \mathrm{~mol} \%$ BMDG were added to the formulations as photoinitiator. For the composite formulations $1 \mathrm{wt} \%$ of BMDG was added.

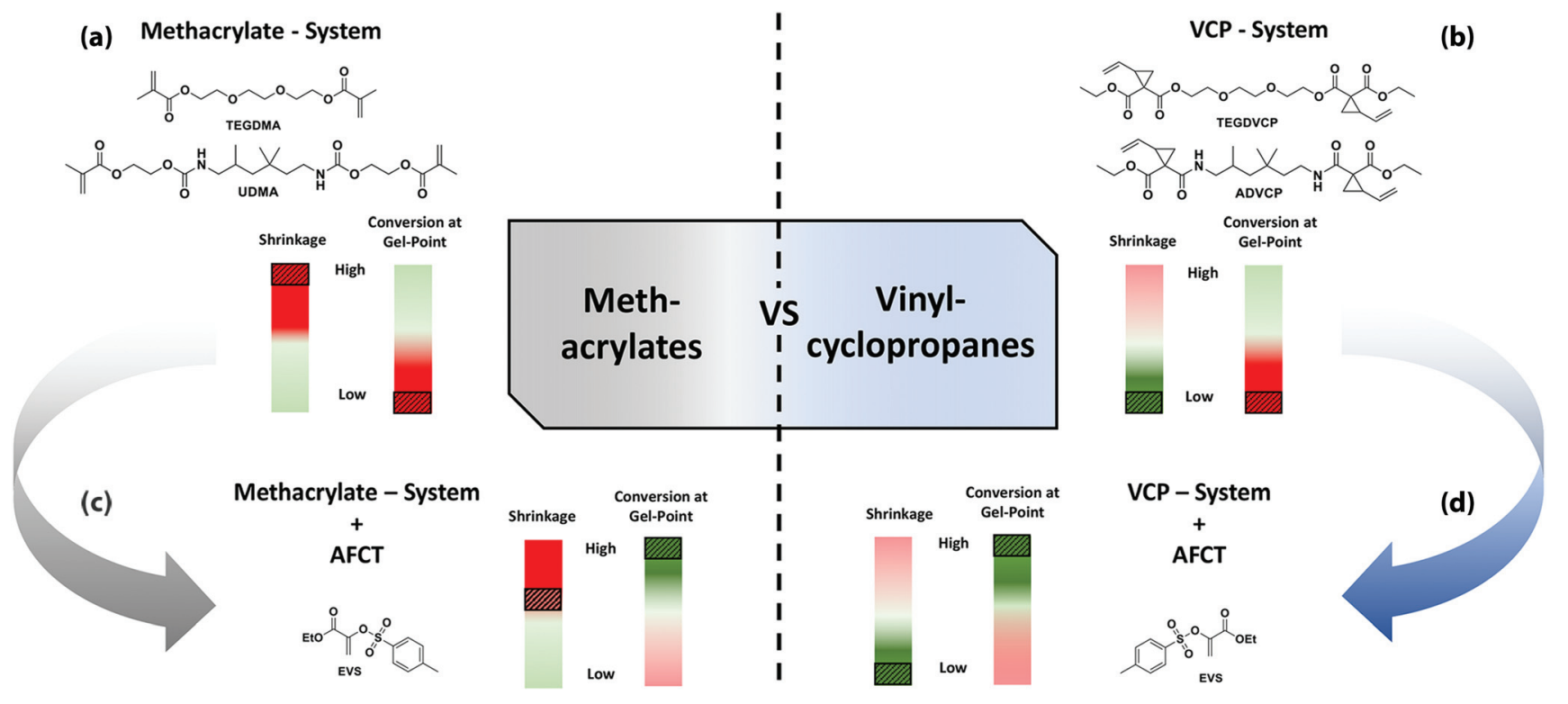

Fig. 2 Illustrations of the benefits from vinylcyclopropanes compared to methacrylates with regards to shrinkage, conversion at gel point and the influence of a chain transfer agent. 


\section{Photo-reactor}

Photo-reactor measurements were performed in a $5 \mathrm{~mL}$ round bottom flask. As an irradiation source an Exfo OmniCureTM 2000 device with a broadband Hg-lamp (300 s, 400-500 nm, $1 \mathrm{~W} \mathrm{~cm}^{-2}$ at the tip of the light guide, $\sim 8 \mathrm{~mW} \mathrm{~cm}^{-2}$ on the surface of the sample measured with an Ocean Optics USB $2000+$ spectrometer) was used. For the experiment $1.14 \mathrm{~g}$ of diethyl 2-vinylcyclopropane-1,1-dicarboxylate (DVD) were weighed in the flask, $0.36 \mathrm{~g}$ of EVS (20 DB\%) and $13.4 \mathrm{mg}$ of BMDG were added and as a solvent $2 \mathrm{~mL}$ deuterated benzene was used. The solution was irradiated, and samples for ${ }^{1} \mathrm{H}$-NMR spectroscopy were taken after 0, 10, 30, 40, 80, 120, 200, 400, $800 \mathrm{~s}$ of irradiation. The double bond conversions (DBCs) of both, the VCP monomer (DVD) and the AFCT reagent (EVS), were determined by integrating the corresponding ${ }^{1} \mathrm{H}$ NMR double bond signals at start and after the respective irradiation periods.

\section{Photo-DSC}

Photo-DSC measurements were conducted on a Netzsch DSC 204 F1 with autosampler. All measurements were performed isocratic at $25{ }^{\circ} \mathrm{C}$ under $\mathrm{N}_{2}$ atmosphere. Monomer formulations (11 $\pm 1 \mathrm{mg}$ ) were irradiated twice with filtered UV/ Visible light (400-500 nm) via an Exfo OmniCureTM series 2000 broadband $\mathrm{Hg}$-lamp under constant $\mathrm{N}_{2}$ flow $\left(20 \mathrm{~mL} \mathrm{~min}^{-1}\right)$. The light intensity was set to $1 \mathrm{~W} \mathrm{~cm}^{-2}$ at the tip of the light guide corresponding to $\sim 20 \mathrm{~mW} \mathrm{~cm} \mathrm{~cm}^{-2}$ on the surface of the sample. The heat flow of the polymerization reaction was recorded as a function of time. The times when the maximum of heat evolution was reached $\left(t_{\max }\right)$ and when $95 \%$ of the overall heat was evolved $\left(t_{95 \%}\right)$ were determined.

\section{RT-NIR-photorheology}

An Anton Paar MCR 302 WESP rheometer with a P-PTD 200/ GL Peltier glass plate, an H-PTD 200 heating hood and a disposable PP25 measuring system was used to conduct the RT-NIRphotorheology experiments. Additionally, the rheometer is coupled with a Bruker Vertex 80 FTIR spectrometer to analyze the chemical conversion of the sample over time. Details for the setup and the measurement procedure are described in literature. $^{31}$ For each measurement, an exact amount of monomer formulation $(130 \mu \mathrm{l})$ was placed at the center of the glass plate and the measurements were conducted at $25{ }^{\circ} \mathrm{C}$ with a gap of $200 \mu \mathrm{m}$. A 5 min acclimatization time was conducted before starting the measurement. The formulations were oscillated with a strain of $1 \%$ and a frequency of $1 \mathrm{~Hz}$. UV/Visible light was used to initiate the reaction, which was emitted via an Exfo OmniCureTM 2000 device with a broadband Hg-lamp (300 s, 400-500 nm, $1 \mathrm{~W} \mathrm{~cm}^{-2}$ at the tip of the light guide, $\sim 8 \mathrm{~mW} \mathrm{~cm}^{-2}$ on the surface of the sample measured with an Ocean Optics USB 2000+ spectrometer). The methacrylate and vinylcyclopropane double bond conversions (DBC) were determined by recording a set of single spectra (time interval $\sim 0.26 \mathrm{~s}$ ) with an OPUS 7.0 software and then integrating the respective double bond bands at $\sim 6160 \mathrm{~cm}^{-1}$
(MA), $\sim 6130 \mathrm{~cm}^{-1}$ (VCP) and $\sim 6150 \mathrm{~cm}^{-1}$ (EVS). The ratio of the double bond peak area at the start and the end of the measurement gave the DBC. All measurements were performed in triplicate with satisfactory reproducibility. ${ }^{4}$

\section{Storage stability}

Storage stability was measured via ${ }^{1} \mathrm{H}-\mathrm{NMR}$ spectroscopy and RT-NIR-photorheology. The mixture VCP/EVS_5 was analyzed via ${ }^{1} \mathrm{H}$-NMR spectroscopy right after the mixture has been formulated $(t=0)$ and again after eight weeks. Additionally, the reactivity of the mixture was measured with RT-NIR-photorheology at $t=0$ and again after eight weeks.

\section{Dynamic mechanical thermal analysis (DMTA)}

The DMTA samples (rectangular-shaped $5 \times 2 \times 40 \mathrm{~mm}^{3}$ ) were prepared in silicon molds and cured with in a Lumamat ${ }^{\circledR} 100$ light oven (400-500 nm, $2 \times 10 \mathrm{~min}$, with 6 Osram Dulux L Blue $18 \mathrm{~W}$ Lamps, total irradiation: $\sim 20 \mathrm{~mW} \mathrm{~cm}^{-2}$ ) for $2 \times$ $10 \mathrm{~min}$ (10 min on each side). An Anton Paar MCR 301 device with a CTD 450 oven and an SRF 12 measuring system was used to perform the DMTA measurements. The prepared DMTA samples were tested in torsion mode with a frequency of $1 \mathrm{~Hz}$ and a strain of $0.1 \%$. The temperature was increased from -100 to $200{ }^{\circ} \mathrm{C}$ with a heating rate of $2{ }^{\circ} \mathrm{C} \mathrm{min}^{-1}$. The glass transition temperature was defined as the temperature at the maximum dissipation factor $(\tan \delta)$.

\section{Tensile tests}

The tensile test specimens (dumbbell-shaped with a total length of $35 \mathrm{~mm}$ and a parallel constriction region dimension of $2 \times 2 \times 12 \mathrm{~mm}^{3}$ ) were prepared analogously to the DMTA samples. A Zwick Z050 equipped with a $1 \mathrm{kN}$ load cell was used to conduct tensile tests. Five specimens per sample were measured. The specimens were fixed between two clamps and strained with a traverse speed of $5 \mathrm{~mm} \mathrm{~min}^{-1}$. A stress-strain plot was recorded simultaneously.

\section{Composite formulations}

For the composite formulation $7 \mathrm{~g}$ of the monomer mixture (ADVCP/TEGDVCP 7/3 molar ratio), which contained $1 \mathrm{wt} \%$ BMDG, were mixed with $13 \mathrm{~g}$ filler. Then, the filler (PU Glasfüller GM27884 $1 \mu \mathrm{m}$ sil.) was added to the prepared monomer mixture and the final composites were mixed in a three-roll mill (Exakt Apparatebau, Norderstedt, Germany). The methacrylate reference and the VCP-based mixtures containing EVS were prepared in the same ratios.

\section{Shrinkage force measurement of composite formulations}

Shrinkage force was measured based on a method described by Watts et al. using a BZ2.5/TS1S universal testing machine (Zwick, Germany). ${ }^{32}$ A steel rod (height: $50 \mathrm{~mm}$, diameter: $10 \mathrm{~mm}$ ) with a flat end was fixed to the load cell of the universal testing machine. A glass slide $\left(2.85 \times 30 \times 75 \mathrm{~mm}^{3}\right)$ was placed on the stationary part of the machine framework. Both, the flat surface of the steel rod and the middle of the glass slide were rubbed for $20 \mathrm{~s}$ with a primer (Monobond S, Ivoclar 
Vivadent AG). 3 min after the application of the primer, compressed air was blown on both surfaces. ${ }^{32}$ This step will provide a strong bond between the composite and both surfaces (steel rod and glass slide). The composite was applied on the flat surface of the steel rod. The crosshead was lowered $\left(0.5 \mathrm{~mm} \mathrm{~min}^{-1}\right)$ until a composite layer with $0.8 \mathrm{~mm}$ thickness (between the steel rod and the glass slide) was reached. The excess of material around the steel rod was removed. The composite was subsequently light-cured underneath the glass slide for $10 \mathrm{~s}$ using an LED curing light Bluephase 20i (Ivoclar Vivadent AG) with an irradiation intensity of $\sim 20 \mathrm{~mW} \mathrm{~cm} \mathrm{~cm}^{-2}$ at $25{ }^{\circ} \mathrm{C}$. The shrinkage force was measured as a function of time. Each experiment was repeated six times.

\section{Mechanical properties of composite formulations}

The formulated composites were filled in steel forms and the flexural strength specimens $\left(2 \times 2 \times 25 \mathrm{~mm}^{3}\right)$ were obtained by irradiating the resins with a visible light source (Spectramat SP2 polymerization unit, Ivoclar Vivadent AG) for $2 \times 3 \mathrm{~min}$ (3 min for each side). Mechanical properties were measured after storage at RT for $24 \mathrm{~h}$. The measurements were carried out in three-point bending tests (span: $20 \mathrm{~mm}$ ) with a speed of $0.8 \mathrm{~mm} \mathrm{~min}^{-1}$ using a BZ2.5/TS1S universal testing machine (Zwick, Germany).

\section{Results and discussion}

\section{Formulating vinylcyclopropane-based monomers with vinyl sulfonate ester as AFCT reagent}

To prove this assumption, we synthesized vinylcyclopropanebased UDMA and TEGDMA replacement structures. The diethyl 1,1'-(((2,2,4-trimethylhexane-1,6-diyl)bis(azanediyl))-bis (carbonyl))bis(2-vinylcyclopropane-1-carboxylate) (ADVCP) and the O'1,O1-((ethane-1,2-diylbis(oxy))-bis(ethane-2,1-diyl)) 1,1'diethyl bis(2-vinylcyclopropane-1,1-dicarboxylate) (TEGDVCP) have been synthesized according to literature (see also ESI $\dagger$ ). ${ }^{15,16}$ They have been mixed in a 7/3 molar ratio and 0.5 mol\% BMDG was added as photoinitiator. To the mixture different amounts of the vinyl sulfonate ester EVS were added as AFCT reagent. Altogether, six formulations have been prepared in order to test the described concept for shrinkage stress reduction: a methacrylate reference (MA) containing UDMA and TEGDMA in a $7 / 3$ molar ratio, a VCP reference (VCP), and 4 mixtures with the AFCT reagent EVS (VCP/EVS 5, 10, 15 and $20 \mathrm{DB} \%, \mathrm{DB} \%$ is the percentage of AFCT-based double bonds in the resin).

\section{Reactivity of vinylcyclopropane-based monomers in combination with vinyl sulfonate ester}

Photoreactor. Prior to investigation of crosslinking photopolymer resins, a monofunctional VCP/EVS system has been investigated to assess the coreactivity of the AFCT reagent within a VCP-based resin. A formulation of the monofunctional vinylcyclopropane DVD with EVS as AFCT reagent (20 DB\%) was prepared within a photoreactor using deuterated benzene as solvent and BMDG as photointiator. The formu- lation was then irradiated (broadband Hg-lamp, $300 \mathrm{~s}$, $400-500 \mathrm{~nm}, \sim 8 \mathrm{~mW} \mathrm{~cm} \mathrm{~cm}^{-2}$ on the surface of the sample) and samples for ${ }^{1} \mathrm{H}$ NMR spectroscopy measurements were taken after set time intervals. It was shown that EVS is consumed uniformly with the VCP monomer (Fig. S1 $\dagger$ ). With respect to these results, a regular copolymerization can be assumed for crosslinking system.

Photo-DSC. In order to evaluate the reactivity of the prepared monomer formulations, photo-DSC experiments were performed at $25{ }^{\circ} \mathrm{C}$ under nitrogen atmosphere and the evolved heat of polymerization upon irradiation was measured. The effect of EVS on the photoreactivity of a VCP-system was evaluated and additionally the formulations were compared to the respective methacrylate reference without chain transfer agent. The time until the maximum rate of polymerization is reached $\left(t_{\max }\right)$ is a measure for the speed at the beginning of the curing reaction. Moreover, the time where $95 \%$ of the reaction heat had been developed $\left(t_{95 \%}\right)$, can be seen as a measure for the overall speed and progress of the photopolymerization reaction. Most commonly, AFCT reagents are known to cause retardation within polymerization reactions. ${ }^{23}$ However, EVS is known for its high reactivity with methacrylates ${ }^{29}$ and the photo-DSC study showed no significant decrease of the reactivity with the addition of EVS (Fig. S2 $\uparrow$ ) for vinylcyclopropanes. At low concentrations of EVS the reaction is slightly slower with a $t_{\max }$ at $4.9 \mathrm{~s}$ compared to $4.3 \mathrm{~s}$ for the VCP reference. However, by increasing the concentration of the AFCT reagent, the polymerization reaction becomes faster $\left(t_{\max }=4 \mathrm{~s}\right)$ compared to the VCP reference. A similar effect could be observed for the $t_{95 \%}$ values, which showed a slight retardation at the lowest concentration of EVS ( $t_{95 \%}$ of VCP/EVS_5 is $\left.22.9 \mathrm{~s}\right)$, but with the increase of EVS $t_{95} \%$ for VCP/EVS_20 reached a value of $18.1 \mathrm{~s}$. This is significantly faster compared to the VCP reference $(21.1 \mathrm{~s})$. The mixture with $20 \mathrm{DB} \%$ EVS had a slightly higher $t_{\max }(4 \mathrm{~s})$ but a lower $t_{95} \%(18.1 \mathrm{~s})$ value than the methacrylate-based reference $\left(t_{\max }\right.$ of $2.6 \mathrm{~s}, t_{95 \%}$ of $\left.23.3 \mathrm{~s}\right)$ (Table $\mathrm{S} 1 \dagger$ ) and this further proves the surprisingly high reactivity of EVS with vinylcyclopropanes.

The photo-DSC plot consists of only one peak for every formulation, thus overall reaction heat is detected preventing the differentiation of the heat contribution to the reaction for each reactive species (e.g. VCP or EVS). Therefore, calculating the double bond conversion (DBC) via the recorded heat of polymerization can only be achieved with the pure MA (65\%, $54.8 \mathrm{~kJ} \mathrm{~mol}^{-1}$ for a monofunctional MA giving a theoretical heat for the MA mixture of $\left.263.9 \mathrm{~J} \mathrm{~g}^{-1}\right)^{33}$ and VCP resins $(58 \%$, $98 \mathrm{~kJ} \mathrm{~mol}^{-1}$ for a monofunctional VCP giving a theoretical heat for the VCP mixture of $401.5 \mathrm{~J} \mathrm{~g}^{-1}$ ). ${ }^{34}$ However, as stated in the photoreactor studies of the monofunctional VCP/EVS system a good coreactivity of EVS with crosslinking VCPs can be assumed. Furthermore, the increased polymerization heat for EVS-based formulations (277-316 $\mathrm{J} \mathrm{g}^{-1}$ ) compared to the pure VCP resin $\left(234.7 \mathrm{~J} \mathrm{~g}^{-1}\right)$ is a strong indication for improved conversion as AFCT reagents are known for low and sometimes even no reaction heat due to the balance of bond breakage and bond formation. ${ }^{26}$ 
RT-NIR-photorheology. RT-NIR-photorheology is another excellent method to track a polymerization process in situ via rheology (storage modulus $\mathrm{G}^{\prime}$ from oscillatory measurements and normal force $\mathrm{F}_{\mathrm{N}}$ indicating shrinkage stress, time to gelation $t_{\text {gel }}$ ) as well as the conversions of the monomer via NIR spectroscopy $\left(\mathrm{DBC}_{\text {gel }}\right.$ and $\left.\mathrm{DBC}_{\text {final }}\right) \cdot{ }^{31}$ With these experiments the effectiveness of EVS during the photopolymerization in VCP-based formulations could be confirmed. Both reference mixtures, purely MA or VCP-based, have an occurring shrinkage stress immediately after the photopolymerization has started. At conversions of approximately $45 \%$ the methacrylic reference suffers already from the same shrinkage stress as the VCP reference does at its final conversion (73\%) (Fig. 3a). The formulations containing EVS show a marginal shrinkage stress beginning at low conversions. The normal force value stays under $2 \mathrm{~N}$ until a double bond conversion of $\sim 30-55 \%$ is reached, which corresponds to the conversion at the gel point $\left(\mathrm{DBC}_{\mathrm{gel}}\right)$. After vitrification the shrinkage stress starts to evolve and reaches about the same value as the VCP reference but a higher final DBC could be observed for all VCP/EVS formu-

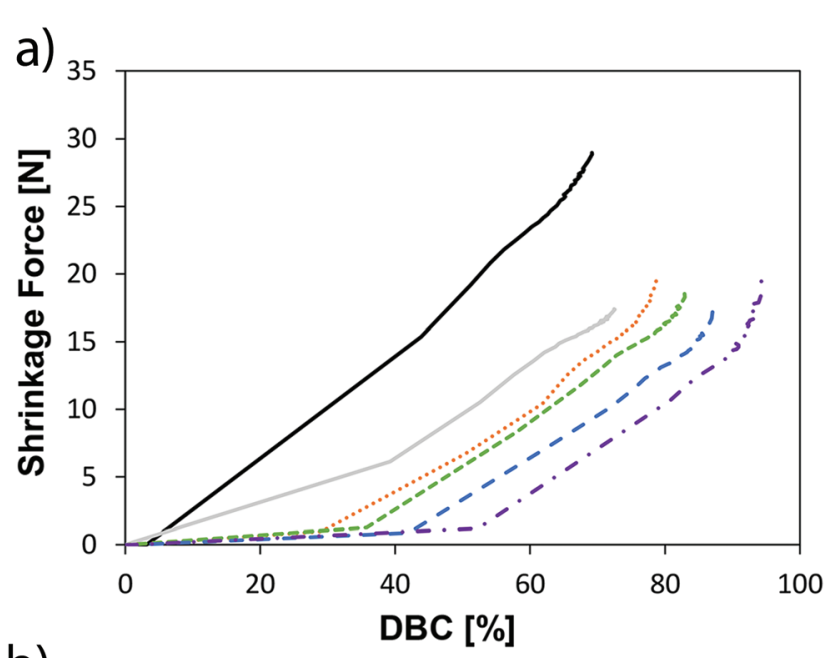

b)

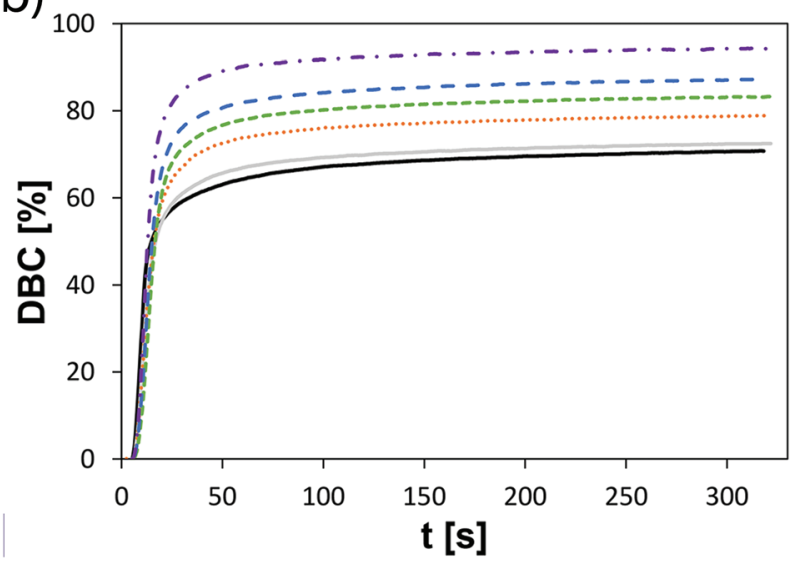

Fig. 3 RT-NIR-photorheology of all six tested resins; (a) shrinkage force over DBC; (b) DBC over time. MA (solid, black); VCP (solid, grey), VCP/EVS_5 (dot), VCP/EVS_10 (short dash), VCP/EVS_15 (long dash), VCP/EVS_20 (dash dot); light source: Omnicure 2000 with 400-500 nm filter, intensity $\sim 20 \mathrm{~mW} \mathrm{~cm}^{-2}$ at $25^{\circ} \mathrm{C}$. lations (Fig. 3b). The final DBC for the MA and the VCP reference derived from RT-NIR-photorheology is slightly higher than in the photo DSC study. Nevertheless, the trend of comparable final conversion is upheld $(\sim 60 \%$ in photo-DSC and $\sim 70 \%$ in RT-NIR-photorheology. The formulation VCP/EVS_15 exhibits the lowest normal force of all EVS-based resins $(18.4 \mathrm{~N})$ and was just slightly higher than the normal force for the VCP reference $(17.5 \mathrm{~N})$ (Fig. S3, Table S2†). By stopping the reactions at a conversion of 70\% (MA: 27.2 N, VCP: 16.28 N), which correlates to the $\mathrm{DBC}_{\text {final }}$ of the $\mathrm{MA}$ reference, the shrinkage stress could be decreased even further (VCP/EVS_20: $7.95 \mathrm{~N}$ ). The measured normal force of all VCP mixtures was significantly lower than the value for the MA reference $(27.3 \mathrm{~N})$. The mixture with $20 \mathrm{DB} \%$ EVS reaches a conversion of over $90 \%$, which is more than $20 \%$ higher than both reference mixtures (Fig. 3b) and further strengthens the postulation for uniform consumption and proves good coreactivity for EVS in VCP-based formulations. The combination of VCPs with EVS also shows perfectly how the gel point is shifted toward higher conversions compared to the references. The homogenous integration of the EVS in the formed network leads to a shift of the gel point. This regular consumption of EVS leads to shorter chains and a longer mobility of the formed network. In this case, the new network reaches the gel point at a later stage of the reaction with the result that the propagation can continue to a greater extent. In addition, this leads to the conclusion why higher overall conversions are reached, keeping in mind that VCP and EVS conversion cannot be differentiated in this case due to overlapping NIR signals. Through the mentioned shift of the gel point, the radicals have more time to attack new double bonds and propagate, thus consuming a higher percentage of the total available double bonds in the system. The storage modulus $\mathrm{G}^{\prime}$ could also be increased compared to the VCP reference (0.84 MPa). The VCP/EVS_20 resin reached the same value as the $\mathrm{MA}$ reference $(1.07 \mathrm{MPa})$ (Fig. S4 $\uparrow$ ). With the combination of EVS and VCP it was possible to generate a polymer network with increased double bond conversions and even further reduced shrinkage stress.

Additionally, good storage stability for the VCP/EVS_5based formulation over a storage period of 8 weeks at room temperature has been confirmed via ${ }^{1} \mathrm{H}$ NMR spectroscopy (Fig. S5 $\dagger$ ). After eight weeks neither polymerization nor decomposition of the components in the formulation took place. Subsequent RT-NIR-photorheology tests of the stored formulations showed no deterioration in reactivity compared to the initial formulation. Conversion (Fig. S6†) as well as storage modulus plots (Fig. S7†) are well matched. This is of particular interest when thinking about industrial application of the developed resin systems.

\section{(Thermo)mechanical and mechanical properties of final photopolymer networks}

Dynamic mechanical thermal analysis (DMTA). The thermomechanical properties within the fabricated photopolymer networks were investigated via DMTA. A crucial characteristic for homogeneous polymer networks is their thermomechanical 
behavior and the underlying thermal polymer phase transitions. While inhomogeneous methacrylate photopolymer networks tend to exhibit rather broad thermal polymer phase transitions, very defined and sharp transitions can be expected for VCP photopolymers combined with EVS. They should exhibit more homogeneous and defined network architectures.

All prepared photopolymerizable formulations were cast into silicone molds, photopolymerized in a Lumamat ${ }^{\circledR} 100$ (400-500 nm, $2 \times 10 \mathrm{~min}$, with 6 Osram Dulux L Blue $18 \mathrm{~W}$ Lamps, total irradiation: $\sim 20 \mathrm{~mW} \mathrm{~cm}^{-2}$ ) and characterized by DMTA in a temperature region of -100 to $200{ }^{\circ} \mathrm{C}$. The storage modulus (G', Fig. 4a) and loss factor ( $\tan \delta$, Fig. $4 \mathrm{~b})$ plots of the newly synthesized VCP networks were studied in comparison to the unregulated methacrylate matrix polyMA. The storage modulus $\mathrm{G}^{\prime}$ increased with a higher amount of EVS, which can be explained by the significantly improved conversion $(>80 \%)$ and is another indication for a good coreactivity of EVS with VCPs. The poly/EVS_15 had a $\mathrm{G}_{37}^{\prime}{ }^{\circ} \mathrm{C}$ of $1160 \mathrm{MPa}$ which was significantly higher than the reference polyVCP (878 MPa) and just slightly lower than polyMA (1520 MPa). The DMTA measurements showed also that the width of the peak of $\tan \delta$ gets sharper with increasing concentration of EVS. PolyVCP/EVS_20 had a lower value for the full width at half maximum of the $\tan \delta$ peak ( fwhm $=26^{\circ} \mathrm{C}$ ) than the references polyVCP $\left(\right.$ fwhm $\left.=29^{\circ} \mathrm{C}\right)$ and polyMA $\left(101^{\circ} \mathrm{C}\right)$ reference. The glass transition temperature $T_{\mathrm{g}}$ also decreased from $91{ }^{\circ} \mathrm{C}$ (polyVCP) with the increase of the EVS to $68{ }^{\circ} \mathrm{C}$ (polyVCP/ EVS_20) (Fig. 4b; Table S3†). Again a lower value than for the reference polymer networks could be observed. The photopolymers with EVS reached a lower $T_{\mathrm{g}}$ with a more narrow glass transition indicating a much more homogeneous and tailored photopolymer network architecture. A reduced crosslinking density is indicated for EVS-based polymers by the lower storage modulus at temperatures above $T_{\mathrm{g}}\left(G_{\mathrm{r}}^{\prime}-\right.$ storage modulus at the rubbery state).

Tensile test. Conducted tensile tests of the fabricated polymers were compared to the unregulated reference networks (polyMA and polyVCP). An increase of the strain and stress in combination with the AFCT reagent EVS was observed (Fig. 4c). A higher strain could be observed with poly/EVS_15, which yielded $6.8 \%$ in strain at break compared to only $3.8 \%$ of the reference polymer polyMA and $5.7 \%$ compared to the polyVCP. The tensile strength was significantly improved to 57 MPa for polyVCP/EVS_20 compared to the polyVCP reference (47 MPa). The measurement errors are in an acceptable range (Table S4†). Therefore, it can be concluded that the addition of EVS has a positive effect on both, the tensile strength and strain at break, and the VCP/EVS polymers almost reach the tensile strength of the methacrylate reference polyMA, while exhibiting higher strain at break (Table S4†े).

\section{Composite formulations}

Because of the high reactivity and the enhanced reduction of shrinkage stress combined with the high double bond conversion, those materials offer great potential for dental appli-
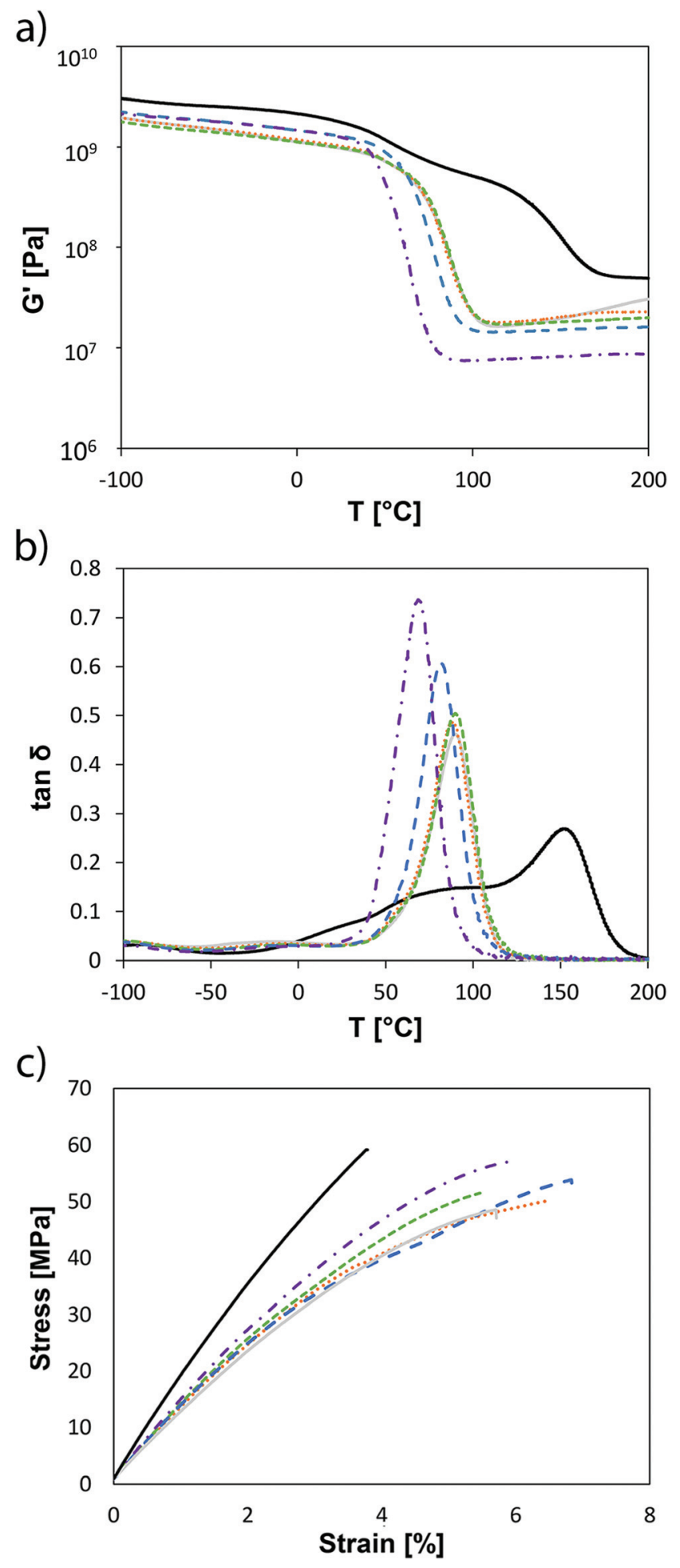

Fig. 4 DMTA (a - storage modulus over temperature, b - $\tan \delta$ over temperature) $\&$ tensile test measurements (c - stress over strain) with polyMA (solid, black); polyVCP (solid, grey), polyVCP/EVS_5 (dot), polyVCP/EVS_10 (short dash), polyVCP/EVS_15 (long dash), polyVCP/ EVS_20 (dash dot); light source: Lumamat ${ }^{\circledR} 100$ light oven, $400-500 \mathrm{~nm}$ filter, intensity $\sim 20 \mathrm{~mW} \mathrm{~cm}{ }^{-2}$ at $25^{\circ} \mathrm{C}$, curing time: $2 \times 10 \mathrm{~min}$. 
cations. Therefore, further evaluation of the materials was conducted by formulating dental composites. Dental composites have become more common as restorative material over the last decades. ${ }^{31,32}$ They present excellent aesthetic and mechanical properties and are easy to apply. ${ }^{35}$ They are based on an organic and inorganic matrix. The organic matrix is typically a mixture of crosslinking dimethacrylates and additives (e.g. photoinitiator systems, stabilizers, pigments, adhesives). The inorganic matrix is mainly based on silanized glass fillers. Two of the most commonly used crosslinking monomers are bis-[(2-methacryloyloxyethoxy-carbonyl)-amino]-2,2,4-trimethylhexane (UDMA) and 2-[2-[2-(2-methylprop-2-enoyloxy)ethoxy] ethoxy]ethyl 2-methylprop-2-enoate (TEGDMA). ${ }^{31,32}$ Methacrylatebased composites tend to have excellent mechanical and esthetic properties but the major drawback is the polymerization-induced shrinkage stress. The composites were formulated with $13 \mathrm{~g}$ silanized Ba-Al glass filler (GM 27884, Company Schott) with a particle size of $1 \mu \mathrm{m}, 7 \mathrm{~g}$ monomer mixture and $1 \mathrm{wt} \%$ of BMDG.

Shrinkage force measurements of composite formulations. Shrinkage force was measured based on a method described by Watts using a BZ2.5/TS1S universal testing machine (Zwick, Germany). ${ }^{32}$ The composite was subsequently light-cured underneath a glass slide for $10 \mathrm{~s}$ using an LED curing light Bluephase 20i (Ivoclar Vivadent AG). The shrinkage force was measured as a function of time. The shrinkage force measurement of the composite showed similar values for the mixtures polyVCP/EVS_5, 10, 15 and 20 (Fig. 5a). They exhibit maximum shrinkage values of approximately $45 \mathrm{~N}$ which was about the same as for the VCP reference with $47 \mathrm{~N}$. The composite with polyVCP/EVS_20 was able to reduce the shrinkage stress to 42 $\mathrm{N}$, which is significantly lower than the composites containing polyMA (55 N) and the polyVCP $(47 \mathrm{~N})$ reference. In comparison to the composite containing the polyMA, all polyVCP/EVS samples showed significantly reduced shrinkage force (Table S5†).

Mechanical properties of composite formulations. Additionally, the flexural strength and flexural modulus of the prepared composites have been measured. The measurements showed how the E-modulus increases from $6600 \mathrm{MPa}$ (polyVCP) to $7277 \mathrm{MPa}$ (polyVCP/EVS_20) with the addition of AFCT reagent and the flexural strength stayed in the same range (112 N polyVCP, $108 \mathrm{~N}$ polyVCP/EVS_20). The values for the E-modulus and the flexural strength were slightly lower compared to the methacrylate reference polyMA, but there was no deterioration noticed in comparison with the reference polyVCP (Fig. 5b, Table S6†).

\section{Conclusion}

In conclusion, we have presented a VCP-based monomer mixture in combination with an ester-activated vinyl sulfonate ester EVS as AFCT reagent, which yields an enhanced reduction of the polymerization-induced shrinkage stress via radical ring opening polymerization and combined shifting of
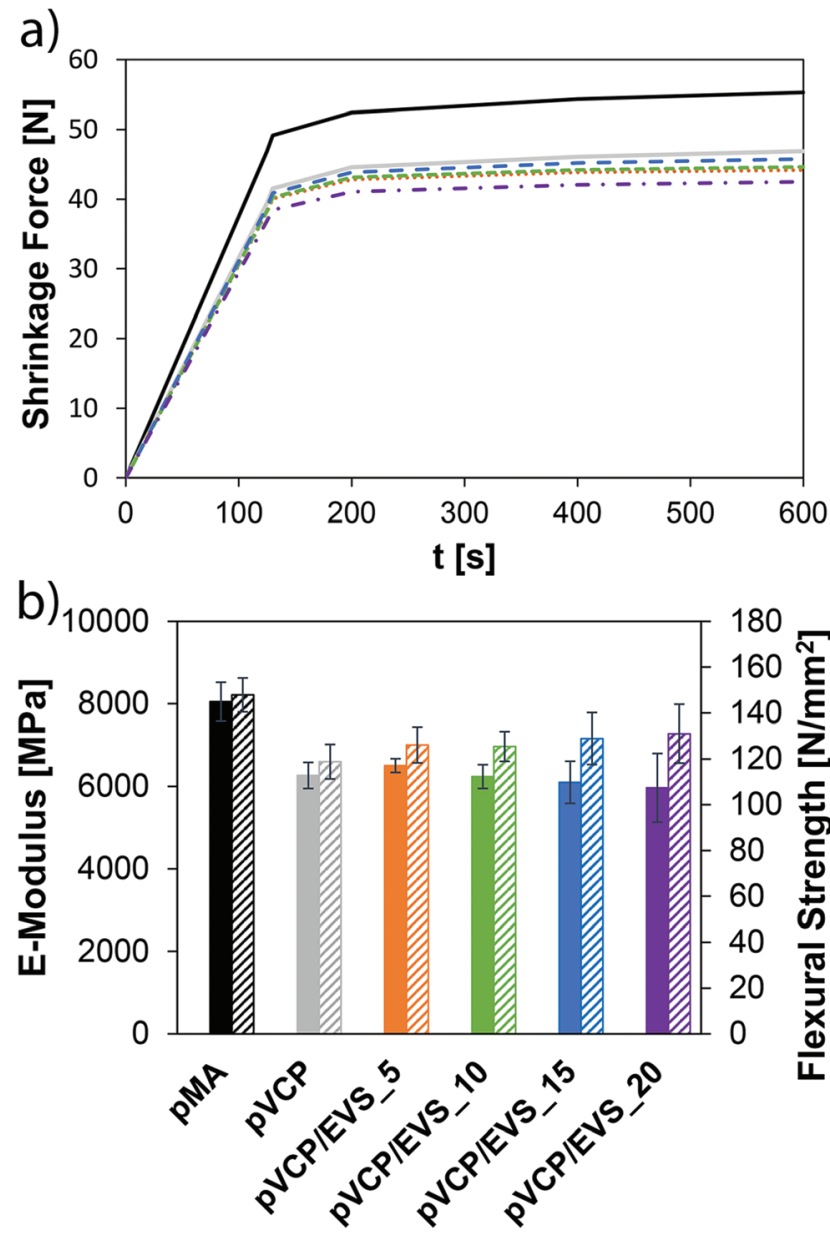

Fig. 5 Shrinkage force plots (a, light source: Bluephase 20i by Ivoclar Vivadent $A G$, intensity $\sim 20 \mathrm{~mW} \mathrm{~cm}{ }^{-2}$ at $25^{\circ} \mathrm{C}$ ) of composites with MA (solid, black); VCP (solid, grey), VCP/EVS_5 (dot), VCP/EVS_10 (short dash), VCP/EVS_15 (long dash), VCP/EVS_20 (dash dot), E-modulus (solid fill) and flexural strength (pattern fill) of the formulated composites (b, light source: Spectramat SP2 polymerization unit by Ivoclar Vivadent AG; curing time: $2 \times 3 \mathrm{~min}$ ).

the gel point toward higher conversions. EVS shows good coreactivity in VCP-based formulations and surprisingly no significant influence on reactivity with the increase of the EVS concentration could be observed. Due to both effects (RROP via VCP and network regulation via EVS) more homogenous photopolymer networks with higher overall double bond conversions are obtained. The DMTA measurements showed a shift of the $T_{\mathrm{g}}$ toward lower temperatures and a more narrow peak for $\tan \delta$ with the addition of the AFCT reagent without compromising on storage modulus at application temperature $\left(\mathrm{G}_{37}^{\prime}{ }^{\circ} \mathrm{C}\right)$. The tensile tests showed that the mechanical properties of the monomer mixtures could also be improved with the addition of EVS and are able to compete with a commercially applied methacrylate reference.

The shrinkage force in prepared composites could also be lowered with the VCP/EVS combination and was significantly lower than for the methacrylate reference polyMA. The composites further exhibit a slightly increased E-modulus with the 
addition of EVS in VCP-based matrices and the flexural strength was slightly lowered. The combination of VCPs with EVS gave very promising results, which outline the possibility to compete with the methacrylate pendants. The VCP/EVSbased mixtures showed improved mechanical properties, good storage stability and high reactivity. Most importantly, significantly lower values for shrinkage stress could be obtained while improving overall conversion. With the presented concept the polymerization-induced shrinkage and shrinkage stress can be significantly reduced even further, which hints toward a high potential for the fields of application (e.g. dental materials, lithography-based 3D-printing).

\section{Author contributions}

The manuscript was written through contributions of all authors. All authors have given approval to the final version of the manuscript.

\section{Conflicts of interest}

There are no conflicts to declare.

\section{Acknowledgements}

Funding by the Christian Doppler Research Association and the company Ivoclar Vivadent AG within the framework of a Christian Doppler Laboratory for "Photopolymers in Digital and Restorative Dentistry" and the financial support by the Austria Federal Ministry for Digital and Economic Affairs and the National Foundation for Research, Technology and Development are gratefully acknowledged. The authors also thank Daniel Alonso Cerron Infantes for the design of the TOC graphic.

\section{References}

1 Photoinitiation, Photopolymerization, and Photocuring: Fundamentals and Applications, ed. J.-P. Fouassier, Hanser/ Gardner, 1995.

2 T. Nardi, L. P. Canal, M. Hausmann, F. Dujonc, V. Michaud, J.-A. E. Manson and Y. Leterrier, Prog. Org. Coat., 2015, 87, 204-212.

3 D. Karalekas and A. Aggelopoulos, J. Mater. Process. Technol., 2003, 136, 146-150.

4 X. Kuang, K. Chen, C. K. Dunn, J. Wu, V. C. F. Li and H. J. Qi, ACS Appl. Mater. Interfaces, 2018, 10, 73817388.

5 M. Sangermano, A. Chiolerio, G. Marti and P. Martino, Macromol. Mater. Eng., 2013, 298, 607-611.

6 K. K. Baikerikar and A. B. Scranton, Polymer, 2000, 42, 431441.

7 J. W. Stansbury, Dent. Mater., 2012, 28, 13-22.
8 C. T. W. Meereis, E. A. Munchow, W. L. de Oliveira da Rosa, A. F. da Silva and E. Piva, J. Mech. Behav. Biomed. Mater., 2018, 82, 268-281.

9 R. R. Braga, R. Y. Ballester and J. L. Ferracane, Dent. Mater., 2005, 21, 962-970.

10 J. Han, S. Jiang, Y. Gao and F. Sun, J. Mater. Chem. C, 2016, 4, 10675-10683.

11 R. R. Moraes, J. W. Garcia, M. D. Barros, S. H. Lewis, C. S. Pfeifer, J. Liu and J. W. Stansbury, Dent. Mater., 2011, 27, 509-519.

12 J. W. Stansbury, R. R. Moraes, J. W. Garcia, M. D. Barros, S. H. Lewis and C. S. Pfeifer, Dent. Mater., 2010, 26, e39e40.

13 N. Moszner and U. Salz, Macromol. Mater. Eng., 2007, 292, 245-271.

14 T. T. Fumio Sanda and T. Endo, Macromolecules, 1993, 26, 1818-1824.

15 P. Pineda Contreras, C. Kuttner, A. Fery, U. Stahlschmidt, V. Jerome, R. Freitag and S. Agarwal, Chem. Commun., 2015, 51, 11899-11902.

16 Y. Catel, P. Fässler, U. Fischer, C. Gorsche, S. Schörpf, S. Tauscher, R. Liska and N. Moszner, Macromol. Mater. Eng., 2017, 302, 1700021.

17 C. E. Hoyle and C. N. Bowman, Angew. Chem., Int. Ed., 2010, 49, 1540-1573.

18 R. Hoogenboom, Angew. Chem., Int. Ed., 2010, 49, 34153417.

19 C. G. Andreas Oesterreicher, S. Ayalur-Karunakaran, M. E. Andreas Moser, G. Pinter, S. Schlögl and T. G. Robert Liska, Macromol. Rapid Commun., 2016, 37, 17011706.

20 S. C. L. Paricher Esfandiari, J. J. Lagref, R. Frantz, Z. Cherkaoui and R. Liska, J. Polym. Sci., 2013, 51, 42614266.

21 M. Podgorski, E. Becka, M. Claudino, A. Flores, P. K. Shah, J. W. Stansbury and C. N. Bowman, Dent. Mater., 2015, 31, 1263-1270.

22 M. Podgórski, C. Wang, Y. Yuan, D. Konetski, I. Smalyukh and C. N. Bowman, Chem. Mater., 2016, 28, 5102-5109.

23 G. Moad, E. Rizzardo and S. H. Thang, Polymer, 2008, 49, 1079-1131.

24 Y. Yagci and I. Reetz, React. Funct. Polym., 1999, 42, 255264.

25 W. D. Cook, S. Chausson, F. Chen, L. Le Pluart, C. N. Bowman and T. F. Scott, Polym. Int., 2008, 57, 469478.

26 C. Gorsche, M. Griesser, G. Gescheidt, N. Moszner and R. Liska, Macromolecules, 2014, 47, 7327-7336.

27 C. Gorsche, T. Koch, N. Moszner and R. Liska, Polym. Chem., 2015, 6, 2038-2047.

28 T. F. Scott, A. D. Schneider, W. D. Cook and C. N. Bowman, Science, 2005, 308, 1615-1617.

29 K. Seidler, M. Griesser, M. Kury, R. Harikrishna, P. Dorfinger, T. Koch, A. Svirkova, M. MarchettiDeschmann, J. Stampfl, N. Moszner, C. Gorsche and R. Liska, Angew. Chem., Int. Ed., 2018, 57, 9165-9169. 
30 C. Gorsche, K. Seidler, P. Knaack, P. Dorfinger, T. Koch, J. Stampfl, N. Moszner and R. Liska, Polym. Chem., 2016, 7, 2009-2014.

31 C. Gorsche, R. Harikrishna, S. Baudis, P. Knaack, B. Husar, J. Laeuger, H. Hoffmann and R. Liska, Anal. Chem., 2017, 89, 4958-4968.

32 D. C. Watts, A. S. Mariuf and A. M. Al-Hindia, Dent. Mater., 2003, 19, 1-11.
33 Y. Catel, C. Dellsperger and N. Moszner, Des. Monomers Polym., 2017, 20, 106-117.

34 Y. Catel, U. Fischer, P. Fässler and N. Moszner, Macromol. Chem. Phys., 2016, 217, 2686-2691.

35 K. L. Van Landuyt, J. Snauwaert, J. De Munck, M. Peumans, Y. Yoshida, A. Poitevin, E. Coutinho, K. Suzuki, P. Lambrechts and B. Van Meerbeek, Biomaterials, 2007, 28, 3757-3785. 